\title{
Laboratory Test Result Status
}

National Cancer Institute

\section{Source}

National Cancer Institute. Laboratory Test Result Status. NCI Thesaurus. Code C70768.

A qualifier used to describe the workflow progression of a laboratory observation

outcome in terms of being finished, concluded, interpreted as preliminary, needed to be repeated, etc. 